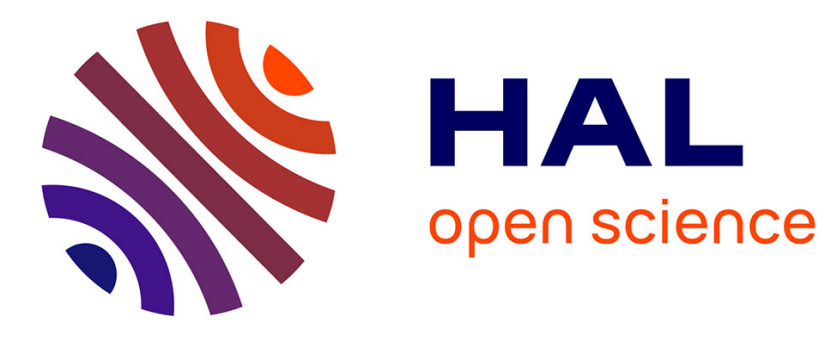

\title{
An equivalent mathematical program for games with random constraints
}

\author{
Vikas Vikram Singh, Abdel Lisser, Monika Arora
}

\section{To cite this version:}

Vikas Vikram Singh, Abdel Lisser, Monika Arora. An equivalent mathematical program for games with random constraints. 2021. hal-03106551

\section{HAL Id: hal-03106551 \\ https://hal-centralesupelec.archives-ouvertes.fr/hal-03106551}

Preprint submitted on 11 Jan 2021

HAL is a multi-disciplinary open access archive for the deposit and dissemination of scientific research documents, whether they are published or not. The documents may come from teaching and research institutions in France or abroad, or from public or private research centers.
L'archive ouverte pluridisciplinaire HAL, est destinée au dépôt et à la diffusion de documents scientifiques de niveau recherche, publiés ou non, émanant des établissements d'enseignement et de recherche français ou étrangers, des laboratoires publics ou privés. 


\title{
An equivalent mathematical program for games with random constraints
}

\author{
Vikas Vikram Singh $^{\mathrm{a}}$, Abdel Lisser ${ }^{\mathrm{b}}$, Monika Arora ${ }^{\mathrm{c}}$ \\ ${ }^{a}$ Department of Mathematics, Indian Institute of Technology Delhi, Hauz Khas, New Delhi, \\ 110016, India. \\ ${ }^{b}$ L2S, CentraleSupélec Université Paris Saclay, 91190 Gif-sur-Yvette, France. \\ ${ }^{c}$ Department of Mathematics, Indraprastha Institute of Information Technology Delhi, New \\ Delhi, 110020, India.
}

\begin{abstract}
We consider an $n$-player chance-constrained game under elliptically symmetric distributions. For a confidence level greater than 0.5 and certain class of payoff functions and strategy sets, we suitably construct an equivalent mathematical program whose global maximizer is a Nash equilibrium.

Keywords: Chance-constrained game, Elliptically symmetric distribution, Mathematical program, Nash equilibrium.
\end{abstract}

\section{Introduction}

The Nash equilibrium is one of the most desired solution concept which is used to study the competition among several selfish and rational players. It is a strategy profile of the players where there is no incentive for unilateral deviation by any player. The theory of games involving Nash equilibrium started with the paper by John Nash [18, where he showed that there exists a mixed strategy Nash equilibrium for finite strategic games. Later it has been shown that a Nash equilibrium of a general non-cooperative game exists under certain conditions on payoff functions and strategy sets of the players [8, 9. The games considered in these papers are deterministic in nature, i.e., the players' strategy

Email addresses: vikassingh@iitd.ac.in (Vikas Vikram Singh),

abdel.lisser@l2s. centralesupelec.fr (Abdel Lisser), monika@iiitd.ac.in (Monika Arora)

Preprint submitted to Elsevier

January 1, 2021 
sets and payoff functions are defined using real valued functions. However, in practical situations the decision making process usually faces various types of uncertainties due to which payoff functions or strategy sets are modeled using random variables [7, 17, 19]. The expected value approach is used to model the uncertainties when the decision makers are risk neutral [20]. For risk averse players, the payoff criterion with the risk measure CVaR [14, 20] and the variance was considered in the literature [6]. Singh et al. [22, 24] considered a finite strategic game where the payoff vector of each player is a random vector. They considered the case where each player is interested in the payoffs that can be obtained with certain confidence. To model this situation, they defined the payoff function of each player using a chance constraint and called such game a chance-constrained game. In [22], the authors showed the existence of a mixed strategy Nash equilibrium for an $n$-player chance-constrained game, when the payoff vector of each player follows a multivariate elliptically symmetric distribution. In [24], the authors proposed an equivalent mathematical program to compute the mixed strategy Nash equilibria of the two player chance-constrained game for elliptically symmetric distributed payoffs. There are some zero-sum chance-constrained games studied in past literature [1, 2, 3, 4].

The chance-constrained games in the above-mentioned papers model the payoffs' uncertainties using chance constraints. However, the uncertainties can be present in the strategy sets due to various external factors. The chanceconstrained based strategy sets are often considered in various applications, e.g., resource constraints in stochastic shortest path problem [5] and risk constraints in portfolio optimization [13] can be modelled using chance constraints. The literature on games with chance-constrained based strategy sets have covered only a small portion of open questions [21, 25]. Singh and Lisser [25] considered a two player zero-sum matrix game where strategy set of each player is defined using individual chance constraints. They showed that the saddle point equilibria of the game can be computed by solving a primal-dual pair of second order cone programs when the random constraint vectors follow multivariate elliptically symmetric distributions. Peng et al. 21] considered an $n$-player game with 
joint chance constraints and showed that there exists a Nash equilibrium of the game if the row vectors are independent and follow multivariate normal distributions. In this paper, we consider an $n$-player game where the strategy sets are defined by individual chance constraints. The random constraint vectors follow multivariate elliptically symmetric distributions. It follows from [12 that an individual chance constraint is equivalent to a second order cone constraint and it makes the feasible strategy set of each player a convex set. Then, under standard quasi-concavity and continuity conditions on the payoff functions, there exists a Nash equilibrium of the game [8, 9]. In order to compute explicitly a Nash equilibrium, we consider a specific payoff function for each player which satisfy the standard conditions. The first term of a player's payoff function is multi-linear in all the players strategies and second term is a quadratic concave function of the player's strategies. Such types of payoff functions are often encountered in practical situations [11, 19, 26, 27]. We propose an equivalent mathematical program for this class of games and show the one-to-one correspondence between a Nash equilibrium of the game and a global maximizer of the mathematical program.

The structure of rest of the paper is as follows. Section 2 contains the definition of a chance-constrained game. Section 3 presents the existence of a Nash equilibrium. The equivalent mathematical program is given in Section 4 .

\section{The model}

We consider an n-player non-cooperative game defined by tuple $\left(I,\left(X^{i}\right)_{i \in I},\left(u_{i}\right)_{i \in I}\right)$, where $I=\{1,2, \ldots, n\}$ is the set of players, $X^{i} \subset \mathbb{R}^{m_{i}}$ is a strategy set of player $i$ and $u_{i}: \prod_{i \in I} X^{i} \rightarrow \mathbb{R}$ is a payoff function of player $i$. The strategy set $X_{i}, i \in I$, is a non-empty, convex and compact set. The product set $X=\prod_{i \in I} X^{i}$ is a set of all strategy profiles of the game. Let $X^{-i}=\prod_{j=1 ; j \neq i}^{n} X^{j}$ be the set of vectors of strategies of all the players but player $i$. The generic elements of $X^{i}, X^{-i}$, and $X$ are denoted by $x^{i}, x^{-i}$, and $x$ respectively. For $y^{i} \in X^{i}$, we define $\left(y^{i}, x^{-i}\right)$ to be a strategy profile where player $i$ chooses a strategy $y^{i}$ and each player $j \in I, j \neq i$, chooses a strategy 
$x^{j}$. We consider the case where the strategies of player $i$ are further restricted by the following random linear constraints

$$
\left(a_{k}^{i}\right)^{T} x^{i} \leq b_{k}^{i}, \quad k=1,2, \ldots, K_{i},
$$

where $a_{k}^{i}$ is an $m_{i} \times 1$ random vector defined on the probability space $(\Omega, \mathcal{F}, \mathbb{P})$ and $b_{k}^{i} \in \mathbb{R} ; T$ denotes the transposition. We consider the case where $k$ th constraint of player $i$ given by 2.1) is satisfied with at least a given probability level $\alpha_{k}^{i}$. Let $\alpha^{i}=\left(\alpha_{k}^{i}\right)_{k=1}^{K_{i}}$ denotes the probability level vector. The chance constraints corresponding to random constraints 2.1 are given by

$$
\mathbb{P}\left\{\left(a_{k}^{i}\right)^{T} x^{i} \leq b_{k}^{i}\right\} \geq \alpha_{k}^{i}, \quad k=1,2, \ldots, K_{i} .
$$

Therefore, for a given probability level vector $\alpha^{i}$, the feasible strategy set of player $i, i \in I$, is defined by

$$
S_{\alpha^{i}}^{i}=\left\{x^{i} \in X^{i} \mid \mathbb{P}\left\{\left(a_{k}^{i}\right)^{T} x^{i} \leq b_{k}^{i}\right\} \geq \alpha_{k}^{i}, \quad k=1,2, \ldots, K_{i}\right\} .
$$

We call the game with payoff functions $\left(u_{i}(\cdot)\right)_{i \in I}$ and strategy sets $\left(S_{\alpha_{i}}^{i}\right)_{i \in I}$ a chance-constrained game. We assume that the set $S_{\alpha^{i}}^{i}, i \in I$, is non-empty, and the probability distribution of the random vector $\left(a_{k}^{i}\right)_{k=1}^{K_{i}}, i \in I$, and the probability level vector $\left(\alpha^{i}\right)_{i \in I}$ are known to all the players. Then, the above chance-constrained game is a non-cooperative game with complete information. For a given strategy profile $x^{-i}$ of other players, a set of best response strategies of player $i$ at probability level vector $\alpha^{i}$ is defined as

$$
B R_{\alpha^{i}}^{i}\left(x^{-i}\right)=\left\{\bar{x}^{i} \in S_{\alpha^{i}}^{i} \mid u_{i}\left(\bar{x}^{i}, x^{-i}\right) \geq u_{i}\left(x^{i}, x^{-i}\right), \forall x^{i} \in S_{\alpha^{i}}^{i}\right\} .
$$

A strategy profile $x^{*}$ is said to be a Nash equilibrium of a chance-constrained game at probability level vector $\left(\alpha^{i}\right)_{i \in I}$ if and only if for each $i \in I$

$$
u_{i}\left(x^{i *}, x^{-i *}\right) \geq u_{i}\left(x^{i}, x^{-i *}\right), \forall x^{i} \in S_{\alpha^{i}}^{i} .
$$

It is clear that $x^{*}$ is a Nash equilibrium if and only if $x^{i *} \in B R_{\alpha^{i}}^{i}\left(x^{-i *}\right)$ for all $i \in$ $I$. The case of two players with zero-sum payoff structure is considered in [25]. The saddle point equilibria of zero-sum chance-constrained games considered 
in 25] can be computed by solving a primal-dual pair of second order cone programs. The $n$-players games where the random constraints 2.1) are jointly satisfied with a given probability are considered in 21. The authors showed the existence of a Nash equilibrium when the random vectors $a_{k}^{i}, k=1,2, \ldots, K_{i}$ are independent and follow a multivariate normal distribution.

\section{Existence of Nash equilibrium}

We consider the case where for each $i \in I$, the vectors $a_{k}^{i}, k=1,2, \ldots, K_{i}$, follow multivariate elliptically symmetric distributions.

Definition 3.1. A d-dimensional random vector $\xi$ follows an elliptically symmetric distribution $\operatorname{Ellip}_{d}(\mu, \Gamma, \varphi)$ if its characteristic function is given by $\mathbb{E} e^{i z^{T} \xi}=e^{i z^{T} \mu} \varphi\left(z^{T} \Gamma z\right)$ where $\varphi$ is the characteristic generator function, $\mu$ is the location parameter, and $\Gamma$ is the positive semidefinite scale matrix.

The class of multivariate elliptically symmetric distributions generalizes the multivariate normal distribution. Some famous distributions belonging to the family of elliptically symmetric distributions include normal distribution with $\varphi(t)=\exp \left\{-\frac{1}{2} t\right\}$, Student's $t$ distribution with $\varphi(t)$ varying with its degree of freedom [15, Cauchy distribution with $\varphi(t)=\exp \{-\sqrt{t}\}$, Laplace distribution with $\varphi(t)=\left(1+\frac{1}{2} t\right)^{-1}$, and logistic distribution with $\varphi(t)=\frac{2 \pi \sqrt{t}}{e^{\pi \sqrt{t}}-e^{-\pi \sqrt{t}}}$ (for details see [10]). It is well known that the family of elliptically symmetric distributions are closed under affine transformations. We summarize this result in Proposition 3.2 .

Proposition 3.2 (Fang et al. [10]). If a d-dimensional random vector $\xi$ follows an elliptically symmetric distribution $\operatorname{Ellip}_{d}(\mu, \Gamma, \varphi)$, then for any $(N \times d)$ matrix $C$ and any $N \times 1$-vector $c, C \xi+c$ follows an $N$-dimensional elliptically symmetric distribution $\operatorname{Ellip}_{N}\left(C \mu+c, C \Gamma C^{T}, \varphi\right)$.

We assume that the random vector $a_{k}^{i}$ follows an elliptically symmetric distribution Ellip $_{m_{i}}\left(\mu_{k}^{i}, \Sigma_{k}^{i}, \varphi_{k}^{i}\right)$, where $\Sigma_{k}^{i}$ is a positive definite scale matrix; 
we denote it by $\Sigma_{k}^{i} \succ 0$. From Proposition 3.2 for a given $x^{i},\left(a_{k}^{i}\right)^{T} x^{i}$ follows Ellip $\left(\left(\mu_{k}^{i}\right)^{T} x^{i},\left(x^{i}\right)^{T} \sum_{k}^{i} x^{i}, \varphi_{k}^{i}\right)$. We can write $\left\|\left(\Sigma_{k}^{i}\right)^{1 / 2} x^{i}\right\|=\sqrt{\left(x^{i}\right)^{T} \sum_{k}^{i} x^{i}}$, where $\left(\Sigma_{k}^{i}\right)^{1 / 2}$ is the unique positive definite square root of matrix $\Sigma_{k}^{i}$ and $\|\cdot\|$ is the Euclidean norm. Then, $\xi_{k}^{i}=\frac{\left(a_{k}^{i}\right)^{T} x^{i}-\left(\mu_{k}^{i}\right)^{T} x^{i}}{\left\|\left(\Sigma_{k}^{i}\right)^{1 / 2} x^{i}\right\|}$ follows a univariate standard elliptically symmetric distributions $\operatorname{Ellip}\left(0,1, \varphi_{k}^{i}\right)$. The positive definite condition on scale matrix is required to get the equivalent second-order cone constraint for individual chance constraint. Therefore, from Lemma 2.2 of [12], the feasible strategy set $S_{\alpha^{i}}^{i}$ can be written as

$S_{\alpha^{i}}^{i}=\left\{x^{i} \in X^{i} \mid\left(x^{i}\right)^{T} \mu_{k}^{i}+\Psi_{\xi_{k}^{i}}^{-1}\left(\alpha_{k}^{i}\right)\left\|\left(\Sigma_{k}^{i}\right)^{\frac{1}{2}} x^{i}\right\| \leq b_{k}^{i}, \forall k=1,2, \ldots, K_{i}\right\}, \quad i \in I$,

where $\Psi_{\xi_{k}^{i}}^{-1}(\cdot)$ is a quantile function of 1-dimensional distribution function induced by the characteristic function $\varphi_{k}^{i}\left(t^{2}\right)$. It is evident that the feasible strategy set $S_{\alpha^{i}}^{i}$ is a compact set. It follows from Proposition 2.1 of [12] that $S_{\alpha^{i}}^{i}$ is a convex set for all $\alpha^{i} \in(0.5,1]^{K_{i}}$ and if each random vector $a_{k}^{i}, k=1,2, \ldots, K_{i}$, has strictly positive density function, $S_{\alpha^{i}}^{i}$ is a convex set for all $\alpha^{i} \in[0.5,1]^{K_{i}}$.

Theorem 3.3. Consider an n-player chance-constrained game defined in Section 2, where the payoff function of player $i, i \in I$, satisfies the following conditions

(i) $u_{i}\left(x^{i}, x^{-i}\right)$ is a quasi-concave function of $x^{i}$ for all $x^{-i} \in X^{-i}$.

(ii) $u_{i}(x)$ is a continuous function of $x$.

Suppose the random vector $a_{k}^{i} \sim \operatorname{Ellip}_{m_{i}}\left(\mu_{k}^{i}, \Sigma_{k}^{i}, \varphi_{k}^{i}\right)$, where $\Sigma_{k}^{i} \succ 0$, for all $k=1,2, \ldots, K_{i}$ and $i \in I$. Then, there exists a Nash equilibrium for all $\alpha \in$ $(0.5,1]^{K_{1}} \times(0.5,1]^{K_{2}} \times \cdots \times(0.5,1]^{K_{n}}$.

Proof. For elliptically symmetric distribution, the feasible strategy sets $S_{\alpha^{i}}^{i}$, $i \in I$, is a non-empty, convex and compact sets for all $\alpha^{i} \in(0.5,1]^{K_{i}}$. Then, under the conditions given in Theorem 3.3 the existence of Nash equilibrium directly follows from [8, 9]. 
The proof of Theorem 3.3 depends on the convexity of feasible strategy sets $S_{\alpha^{i}}^{i}, i \in I$. If $\alpha_{k}^{i}<0.5, k=1,2, \ldots, K_{i}$, under conditions given in Proposition 2.2, $S_{\alpha_{i}}^{i}$ is not a convex set. Note that, in chance constraint programming the confidence levels are typically high, therefore, $\alpha_{k}^{i}>0.5$ is not a very strong requirement in Theorem 3.3 .

\section{Mathematical programming formulation}

We consider a class of $n$-player chance-constrained games which satisfy the conditions $(i)$ and $(i i)$ of Theorem 3.3 . For each $i \in I$, let $J^{i}=\left\{1,2, \cdots, m_{i}\right\}$. Define the product sets $J=\prod_{i \in I} J^{i}$ and $J^{-i}=\prod_{k \in I ; k \neq i} J^{k}$. Consider a vector $\left(r^{i}(s)\right)_{s \in J}$, where $s=\left(s_{1}, s_{2}, \cdots, s_{n}\right)$ with $s_{i} \in J^{i}$. The payoff function of player $i$ is given by

$$
u_{i}(x)=\sum_{s \in J} \prod_{j=1}^{n} r^{i}(s) x_{s_{j}}^{j}-\frac{1}{2}\left(x^{i}\right)^{T} Q_{i} x^{i},
$$

where $Q_{i}$ is a positive definite matrix. The first term of the payoff function of player $i$ is linear in $i$ th player's strategies for a fixed strategy profile of rest of the players and the second term only depends on the strategies of player $i$ and is quadratic in nature. Such a payoff function appears in various applications, e.g., it represents the mean-variance model in portfolio optimization [11, 26, 27. and in electricity market the second term can be the production cost [19] and the first term represents the payoff received due to the interaction among all the players.

For every $s_{i} \in J^{i}$, define $r^{i}\left(s_{i}, x^{-i}\right)=\sum_{s_{-i} \in J^{-i}} \prod_{j \in I ; j \neq i} r^{i}\left(s_{i}, s_{-i}\right) x_{s_{j}}^{j}$. Then, $u_{i}\left(x^{i}, x^{-i}\right)=\sum_{s_{i} \in J^{i}} r^{i}\left(s_{i}, x^{-i}\right) x_{s_{i}}^{i}-\frac{1}{2}\left(x^{i}\right)^{T} Q_{i} x^{i}$. The strategy set $X^{i}$, $i \in I$, is a bounded polyhedron and it is defined as

$$
X^{i}=\left\{x^{i} \in \mathbb{R}^{m_{i}} \mid C^{i} x^{i}=d^{i}, x^{i} \geq \mathbf{0}\right\},
$$

where $C^{i} \in \mathbb{R}^{L_{i} \times m_{i}}, d^{i} \in \mathbb{R}^{L_{i}}$ and $\mathbf{0}$ is an $m^{i} \times 1$ zero vector. In this paper, we identify $\mathbf{0}$ as a zero vector of appropriate dimension. 
Assumption 4.1. For each $i \in I$, the set $S_{\alpha_{i}}^{i}$ is strictly feasible, i.e., there exists an $x^{i} \in \mathbb{R}^{m_{i}}$ such that $x^{i}$ is a feasible point of $S_{\alpha_{i}}^{i}$ and all its inequality constraints are strictly feasible.

The condition given in Assumption 4.1 is a Slater condition which is sufficient for strong duality in convex optimization problem. We use these conditions in order to derive equivalent mathematical program for the chance-constrained game.

\subsection{Best response convex programs}

For a given $x^{-i} \in S_{\alpha^{-i}}^{-i}$, a best response strategy of player $i$ is obtained by solving the following convex optimization problem

$$
\begin{aligned}
& {\left[\mathrm{P}_{i}\right] \max _{x^{i},\left(t_{k}^{i}\right)_{k=1}^{K}} \sum_{s_{i} \in J^{i}} r^{i}\left(s_{i}, x^{-i}\right) x_{s_{i}}^{i}-\frac{1}{2}\left(x^{i}\right)^{T} Q_{i} x^{i}} \\
& \text { s.t } \quad(i) C^{i} x^{i}=d^{i} \\
& \text { (ii) }\left(x^{i}\right)^{T} \mu_{k}^{i}+\Psi_{\xi_{k}^{i}}^{-1}\left(\alpha_{k}^{i}\right)\left\|t_{k}^{i}\right\| \leq b_{k}^{i}, \forall k=1,2, \ldots, K_{i} \\
& (\text { iii }) t_{k}^{i}=\left(\Sigma_{k}^{i}\right)^{\frac{1}{2}} x^{i}, \forall k=1,2, \ldots, K_{i} \\
& \text { (iv) } x^{i} \geq \mathbf{0} .
\end{aligned}
$$

The Lagrangian dual of best response convex optimization problem $\left[\mathrm{P}_{i}\right]$ is given by

$$
\begin{aligned}
\min _{\left(\gamma_{k}^{i}\right)_{k=1}^{K_{i}}, \delta^{i}, \lambda^{i} \geq \mathbf{0}, \beta^{i} \geq \mathbf{0}} & \max _{\left(t_{k}^{i}\right)_{k=1}^{K_{i}}, x^{i} \in \mathbb{R}^{m_{i}}}\left[\sum_{s_{i} \in J^{i}} r^{i}\left(s_{i}, x^{-i}\right) x_{s_{i}}^{i}-\frac{1}{2}\left(x^{i}\right)^{T} Q_{i} x^{i}\right. \\
& +\left(\delta^{i}\right)^{T}\left(d^{i}-C^{i} x^{i}\right)+\sum_{k=1}^{K_{i}}\left(\gamma_{k}^{i}\right)^{T}\left(t_{k}^{i}-\left(\Sigma_{k}^{i}\right)^{\frac{1}{2}} x^{i}\right) \\
+ & \left.\sum_{k=1}^{K_{i}} \lambda_{k}^{i}\left(b_{k}^{i}-\left(x^{i}\right)^{T} \mu_{k}^{i}-\Psi_{\xi_{k}^{i}}^{-1}\left(\alpha_{k}^{i}\right)\left\|t_{k}^{i}\right\|\right)+\left(\beta^{i}\right)^{T} x^{i}\right],
\end{aligned}
$$

where $\delta^{i} \in \mathbb{R}^{L_{i}}$ and $\lambda^{i} \in \mathbb{R}_{+}^{K_{i}}, \gamma_{k}^{i} \in \mathbb{R}^{m_{i}}, k=1,2, \ldots, K_{i}, \beta^{i} \in \mathbb{R}_{+}^{m_{i}}$ are the vectors of Lagrange multipliers corresponding to constraints $(i),(i i),(i i i)$ and $(i v)$, respectively. For a given $x^{-i}$, define a vector $R^{i}\left(x^{-i}\right)=\left(r^{i}\left(s_{i}, x^{-i}\right)\right)_{s_{i} \in J^{i}}$. Then, for a fixed $\left(\gamma_{k}^{i}\right)_{k=1}^{K_{i}}, \delta^{i}$ and $\lambda^{i} \geq \mathbf{0}, \beta^{i} \geq \mathbf{0}$, we have 


$$
\begin{gathered}
\max _{\left(t_{k}^{i}\right)_{k=1}^{K_{i}}, x^{i} \in \mathbb{R}^{m_{i}}}\left[\sum_{s_{i} \in J^{i}} r^{i}\left(s_{i}, x^{-i}\right) x_{s_{i}}^{i}\right)-\frac{1}{2}\left(x^{i}\right)^{T} Q_{i} x^{i}+\left(\delta^{i}\right)^{T}\left(d^{i}-C^{i} x^{i}\right) \\
\left.+\sum_{k=1}^{K_{i}}\left(\gamma_{k}^{i}\right)^{T}\left(t_{k}^{i}-\left(\Sigma_{k}^{i}\right)^{\frac{1}{2}} x^{i}\right)+\sum_{k=1}^{K_{i}} \lambda_{k}^{i}\left(b_{k}^{i}-\left(x^{i}\right)^{T} \mu_{k}^{i}-\Psi_{\xi_{k}^{i}}^{-1}\left(\alpha_{k}^{i}\right)|| t_{k}^{i} \|\right)+\left(\beta^{i}\right)^{T} x^{i}\right] \\
=\max _{x^{i} \in \mathbb{R}^{m_{i}}}\left[-\frac{1}{2}\left(x^{i}\right)^{T} Q_{i} x^{i}+\left(x^{i}\right)^{T}\left(R^{i}\left(x^{-i}\right)-\left(C^{i}\right)^{T} \delta^{i}-\sum_{k=1}^{K_{i}}\left(\Sigma_{k}^{i}\right)^{\frac{1}{2}} \gamma_{k}^{i}\right.\right. \\
\left.\left.-\sum_{k=1}^{K_{i}} \lambda_{k}^{i} \mu_{k}^{i}+\beta^{i}\right)\right]+\max _{\left(t_{k}^{i}\right)_{k=1}^{K_{i}}}\left[\sum_{k=1}^{K_{i}}\left(\left(\gamma_{k}^{i}\right)^{T} t_{k}^{i}-\Psi_{\xi_{k}^{i}}^{-1}\left(\alpha_{k}^{i}\right) \lambda_{k}^{i}\left\|t_{k}^{i}\right\|\right)\right]+\left(\delta^{i}\right)^{T} d^{i}+\sum_{k=1}^{K_{i}} \lambda_{k}^{i} b_{k}^{i}
\end{gathered}
$$

The first max is given by

$$
\frac{1}{2}\left(P_{i}\right)^{T} Q_{i}^{-1} P_{i},
$$

where $P_{i}=R^{i}\left(x^{-i}\right)-\left(C^{i}\right)^{T} \delta^{i}-\sum_{k=1}^{K_{i}}\left(\sum_{k}^{i}\right)^{\frac{1}{2}} \gamma_{k}^{i}-\sum_{k=1}^{K_{i}} \lambda_{k}^{i} \mu_{k}^{i}+\beta^{i}$, and the second max problem is unbounded unless

$$
\left\|\gamma_{k}^{i}\right\| \leq \Psi_{\xi_{k}^{i}}^{-1}\left(\alpha_{k}^{i}\right) \lambda_{k}^{i}, \forall k=1,2, \cdots, K_{i}
$$

Therefore, the dual of $\left[\mathrm{P}_{i}\right]$ is given by

$$
\begin{aligned}
& {\left[\mathrm{D}_{i}\right] \min _{\left(\gamma_{k}^{i}\right)_{k=1}^{K_{i}}, \delta^{i}, \lambda^{i}, \beta^{i}} \frac{1}{2}\left(P_{i}\right)^{T} Q_{i}^{-1} P_{i}+\left(\delta^{i}\right)^{T} d^{i}+\sum_{k=1}^{K_{i}} \lambda_{k}^{i} b_{k}^{i}} \\
& \text { s.t. } \\
& \text { (i) }\left\|\gamma_{k}^{i}\right\| \leq \Psi_{\xi_{k}^{i}}^{-1}\left(\alpha_{k}^{i}\right) \lambda_{k}^{i}, \forall k=1,2, \cdots, K_{i} \\
& \text { (ii) } \lambda^{i} \geq \mathbf{0}, \beta^{i} \geq \mathbf{0} \text {. }
\end{aligned}
$$

\subsection{Mathematical Program}

To the best of our knowledge, the characterization of Nash equilibrium using the global optimal points of a suitably constructed mathematical program was first proposed by Mangasarian and Stone [16. They showed that a Nash equilibrium of a bimatrix game can be obtained from a global maximizer of a quadratic program. Singh et al. [23, 24] characterized the Nash equilibria of the games with random payoffs using the global maximizer of a certain mathematical program. They used the fact that the best response problem of 
each player is equivalent to a primal-dual pair of convex programs for which strong duality holds under Slater conditions. Along the same line, we construct a mathematical program by combining $n$ primal-dual pair $\left[\mathrm{P}_{i}\right]-\left[\mathrm{D}_{i}\right]$ of best response convex programs. Then, we characterize the Nash equilibria of the chance-constrained game using the global optimal points of the mathematical program. Let $\zeta=\left(x^{i},\left(t_{k}^{i}\right)_{k=1}^{K_{i}},\left(\gamma_{k}^{i}\right)_{k=1}^{K_{i}}, \delta^{i}, \lambda^{i}, \beta^{i}\right)_{i \in I}$, and $\phi(\zeta)$ denote the decision variables and objective function of the mathematical program. We have the following characterization.

Theorem 4.2. Consider an n-player chance-constrained game defined in Section 2, where the payoff function $u_{i}(x)$ and the strategy set $X_{i}$ of player $i$, $i \in I$, is given by (4.1) and 4.2), respectively. Suppose the random vectors $a_{k}^{i} \sim$ Ellip $_{m_{i}}\left(\mu_{k}^{i}, \Sigma_{k}^{i}, \varphi_{k}^{i}\right)$, where $\Sigma_{k}^{i} \succ 0$, for all $k=1,2, \ldots, K_{i}$ and $i \in I$. Let Assumption 4.1 holds. Then, for an $\alpha \in(0.5,1]^{K_{1}} \times(0.5,1]^{K_{2}} \times \cdots \times(0.5,1]^{K_{n}}$

1. If $\left(x^{i *}\right)_{i \in I}$ is a Nash equilibrium of the chance-constrained game, there exists a vector $\zeta^{*}=\left(x^{i *},\left(t_{k}^{i *}\right)_{k=1}^{K_{i}},\left(\gamma_{k}^{i *}\right)_{k=1}^{K_{i}}, \delta^{i *}, \lambda^{i *}, \beta^{i *}\right)_{i \in I}$ such that it is a global maximizer of the following mathematical program [MP]

$[\mathrm{MP}]$

$$
\begin{aligned}
\max _{\zeta} \phi(\zeta)=\sum_{i \in I} & {\left[\sum_{s_{i} \in J^{i}} r^{i}\left(s_{i}, x^{-i}\right) x_{s_{i}}^{i}-\frac{1}{2}\left(x^{i}\right)^{T} Q_{i} x^{i}\right.} \\
& \left.-\frac{1}{2}\left(P_{i}\right)^{T} Q_{i}^{-1} P_{i}-\left(\delta^{i}\right)^{T} d^{i}-\sum_{k=1}^{K_{i}} \lambda_{k}^{i} b_{k}^{i}\right]
\end{aligned}
$$

s.t.

(i) $\left\|\gamma_{k}^{i}\right\| \leq \Psi_{\xi_{k}^{i}}^{-1}\left(\alpha_{k}^{i}\right) \lambda_{k}^{i}, \forall k=1,2, \cdots, K_{i}, i \in I$

(ii) $\left(x^{i}\right)^{T} \mu_{k}^{i}+\Psi_{\xi_{k}^{i}}^{-1}\left(\alpha_{k}^{i}\right)\left\|\left(\Sigma_{k}^{i}\right)^{\frac{1}{2}} x^{i}\right\| \leq b_{k}^{i}, \forall k=1,2, \ldots, K_{i}, i \in I$

(iii) $C^{i} x^{i}=d^{i}, i \in I$

(iv) $x^{i} \geq \boldsymbol{O}, \lambda^{i} \geq \boldsymbol{O}, \beta^{i} \geq \boldsymbol{O}, i \in I$.

with objective function value $\phi\left(\zeta^{*}\right)=0$.

2. If $\zeta^{*}=\left(x^{i *},\left(t_{k}^{i *}\right)_{k=1}^{K_{i}},\left(\gamma_{k}^{i *}\right)_{k=1}^{K_{i}}, \delta^{i *}, \lambda^{i *}, \beta^{i *}\right)_{i \in I}$ is a global maximizer of 
the mathematical program [MP], $\left(x^{i *}\right)_{i \in I}$ is a Nash equilibrium of the chance-constrained game.

Proof. 1. Let $\left(x^{i *}\right)_{i \in I}$ be a Nash equilibrium. Then, for each $i \in I$, $\left(x^{i *},\left(t_{k}^{i *}\right)_{k=1}^{K_{i}}\right)$ is an optimal solution of $\left[\mathrm{P}_{i}\right]$ for the fixed $x^{-i *}$. The strong duality holds under Assumption 4.1. Therefore, there exists $\left(\left(\gamma_{k}^{i *}\right)_{k=1}^{K_{i}}, \delta^{i *}, \lambda^{i *}, \beta^{i *}\right)$ which is an optimal solution of $\left[\mathrm{D}_{i}\right]$ such that for each $i \in I$

$$
\sum_{s_{i} \in J^{i}} r^{i}\left(s_{i}, x^{-i *}\right) x_{s_{i}}^{i *}-\frac{1}{2}\left(x^{i *}\right)^{T} Q_{i} x^{i *}=\frac{1}{2} P_{i}^{*} Q_{i}^{-1} P_{i}^{*}+\left(\delta^{i *}\right)^{T} d^{i}+\sum_{k=1}^{K_{i}} \lambda_{k}^{i *} b_{k}^{i},
$$

where $P_{i}^{*}=R^{i}\left(x^{-i *}\right)-\left(C^{i}\right)^{T} \delta^{i *}-\sum_{k=1}^{K_{i}}\left(\sum_{k}^{i}\right)^{\frac{1}{2}} \gamma_{k}^{i *}-\sum_{k=1}^{K_{i}} \lambda_{k}^{i *} \mu_{k}^{i}+\beta^{i *}$. Therefore, $\zeta^{*}=\left(x^{i *},\left(t_{k}^{i *}\right)_{k=1}^{K_{i}},\left(\gamma_{k}^{i *}\right)_{k=1}^{K_{i}}, \delta^{i *}, \lambda^{i *}\right)_{i \in I}$ is a feasible point of [MP] and $\phi\left(\zeta^{*}\right)=0$. Let $\zeta$ be an arbitrary feasible point of [MP]. Then, for each $i \in I,\left(x^{i},\left(t_{k}^{i}\right)_{k=1}^{K_{i}}\right)$ and $\left(\left(\gamma_{k}^{i}\right)_{k=1}^{K_{i}}, \delta^{i}, \lambda^{i}, \beta^{i}\right)$ will be feasible points of $\left[\mathrm{P}_{i}\right]$ and $\left[\mathrm{D}_{i}\right]$, respectively. Then, from weak duality theorem $\phi(\zeta) \leq 0$ for all feasible point $\zeta$. Therefore, $\zeta^{*}$ is a global maximizer of [MP].

2. Let $\zeta^{*}$ be a global maximizer of [MP]. From the proof of first part it follows that $\phi\left(\zeta^{*}\right)=0$. The primal-dual pair $\left[\mathrm{P}_{i}\right]-\left[\mathrm{D}_{i}\right], i \in I$, of convex programs are feasible at $\zeta^{*}$. Therefore, it follows from weak-duality that each part of the objective function is non-negative at $\zeta^{*}$. Hence, 4.3 holds at $\zeta^{*}$. For every $x^{i} \in S_{\alpha^{i}}^{i}$, take $t_{k}^{i}=\left(\Sigma_{k}^{i}\right)^{\frac{1}{2}} x^{i}, k=1,2, \ldots, K_{i}$. Then, $\left(x^{i},\left(t_{k}^{i}\right)_{k=1}^{K_{i}}\right)$ is a feasible solution of $\left[\mathrm{P}_{i}\right]$. Again, from weak duality we have

$$
\sum_{s_{i} \in J^{i}} r^{i}\left(s_{i}, x^{-i *}\right) x_{s_{i}}^{i}-\frac{1}{2}\left(x^{i}\right)^{T} Q_{i} x^{i} \leq \frac{1}{2}\left(P_{i}^{*}\right)^{T} Q_{i}^{-1} P_{i}^{*}+\left(\delta^{i *}\right)^{T} d^{i}+\sum_{k=1}^{K_{i}} \lambda_{k}^{i *} b_{k}^{i},
$$

for all $x^{i} \in S_{\alpha^{i}}^{i}$. From 4.3 , for each $i \in I$, we have

$$
u_{i}\left(x^{i *}, x^{-i *}\right) \geq u_{i}\left(x^{i}, x^{-i *}\right), \forall x^{i} \in S_{\alpha^{i}}^{i} .
$$

Hence, $\left(x^{i *}\right)_{i \in I}$ is a Nash equilibrium of the chance-constrained game. 
The mathematical program $[\mathrm{MP}]$ is a non-convex optimization problem. In general, a non-convex optimization problem is hard to solve. However, the mathematical program $[\mathrm{MP}]$ has a nice structure, e.g., the objective function value is non-positive for all feasible points and a global maximum is attained when the objective function value is zero. Due to these properties, mathematical program $[\mathrm{MP}]$ is not very difficult to solve. The existing nonlinear optimization solvers can be used to compute a global maximizer of [MP]. The similar optimization problems were proposed in [23, 24] and Nash equilibria for randomly generated games of various sizes were computed using fmincon solver in MATLAB. The details about the computation time are given in the numerical sections of [23, 24].

\section{Acknowledgement}

This research was supported by DST/CEFIPRA Project No. IFC/4117/DST-CNRS-5th call/2017-18/2 and CNRS Project No. AR/SB:201807-440.

\section{References}

[1] R. A. Blau, Random-payoff two person zero-sum games, Operations Research 22 (6) (1974) 1243-1251.

[2] R. G. Cassidy, C. A. Field, M. J. L. Kirby, Solution of a satisficing model for random payoff games, Management Science 19 (3) (1972) 266-271.

[3] A. Charnes, M. J. L. Kirby, W. M. Raike, Zero-zero chance-constrained games, Theory of Probability and its Applications 13 (4) (1968) 628-646.

[4] J. Cheng, J. Leung, A. Lisser, Random-payoff two-person zero-sum game with joint chance constraints, European Journal of Operational Research 251 (1) (2016) 213-219.

[5] J. Cheng, A. Lisser, A second-order cone programming approach for linear programs with joint probabilistic constraints, Operations Research Letters 40 (5) (2012) 325-328. 
[6] A. J. Conejo, F. J. Nogales, J. M. Arroyo, R. García-Bertrand, Riskconstrained self-scheduling of a thermal power producer, IEEE Transactions on Power Systems 19 (3) (2004) 1569-1574.

[7] P. Couchman, B. Kouvaritakis, M. Cannon, F. Prashad, Gaming strategy for electric power with random demand, IEEE Transactions on Power Systems 20 (3) (2005) 1283-1292.

[8] G. Debreu, A social equilibrium existence theorem, Proceedings of National Academy of Sciences 38 (1952) 886-893.

[9] K. FAN, Applications oi a theorem concerning sets with convex sections, Mathematische Annalen 163 (1966) 189-203.

[10] K.-T. Fang, S. Kotz, K.-W. Ng, Symmetric Multivariate and Related Distributions, Chapman and Hall, London, New York, 1990.

[11] M. Gilli, D. Maringer, E. Schumann, Numerical Methods and Optimization in Finance, Elsevier, 2011.

[12] R. Henrion, Structural properties of linear probabilistic constraints, Optimization 56 (4) (2007) 425-440.

[13] R. Ji, M. A. Lejeune, Risk-budgeting multi-portfolio optimization with portfolio and marginal risk constraints, Annals of Operations Research 262 (2018) 547-578.

[14] A. Kannan, U. V. Shanbhag, H. M. Kim, Addressing supply-side risk in uncertain power markets: stochastic Nash models, scalable algorithms and error analysis, Optimization Methods and Software 28 (5) (2013) 10951138.

[15] S. Kotz, S. Nadarajah, Multivariate t Distributions and Their Applications, Cambridge University Press, Cambridge, 2004. 
[16] O. L. Mangasarian, H. Stone, Two-person nonzero-sum games and quadratic programming, Journal of Mathematical Analysis and Applications 9 (1964) 348-355.

[17] M. Mazadi, W. D. Rosehart, H. Zareipour, O. P. Malik, M. Oloomi, Impact of wind integration on electricity markets: A chance-constrained Nash Cournot model, International Transactions on Electrical Energy Systems 23 (1) (2013) 83-96.

[18] J. F. Nash, Equilibrium points in n-person games, Proceedings of the National Academy of Sciences 36 (1) (1950) 48-49.

[19] A. Ratha, J. Kazempour, A. Virag, P. Pinson, Exploring market properties of policy-based reserve procurement for power systems, in: 2019 IEEE 58th Conference on Decision and Control (CDC), 2019.

[20] U. Ravat, U. V. Shanbhag, On the characterization of solution sets of smooth and nonsmooth convex stochastic Nash games, SIAM Journal of Optimization 21 (3) (2011) 1168-1199.

[21] P. Shen, V. V. Singh, A. Lisser, General sum games with joint chance constraints, Operations Research Letters 56 (2018) 482-486.

[22] V. V. Singh, O. Jouini, A. Lisser, Existence of Nash equilibrium for chanceconstrained games, Operations Research Letters 44 (5) (2016) 640-644.

[23] V. V. Singh, O. Jouini, A. Lisser, Distributionally robust chanceconstrained games: Existence and characterization of Nash equilibrium, Optimization Letters 11 (7) (2017) 1385-1405.

[24] V. V. Singh, A. Lisser, A characterization of Nash equilibrium for the games with random payoffs, Journal of Optimization Theory and Applications 178 (3) (2018) 998-1013.

[25] V. V. Singh, A. Lisser, A second order cone programming formulation for zero-sum game with chance constraints, European Journal of Operational Research 275 (2019) 839-845. 
[26] M. C. Steinbach, Markowitz revisited: Mean-variance models in financial portfolio analysis, SIAM Reveiw 43 (2001) 31-85.

[27] J. Wang, F. He, X. Shi, Numerical solution of a general interval quadratic programming model for portfolio selection, PLoS ONE 14 (2019) https://doi.org/10.1371/journal.pone.0212913. 